\title{
ADAPTIVE INTERNAL MODEL CONTROL WITH REFERENCE INPUT PREDICTOR FOR TIME-DELAY SYSTEMS
}

\author{
X. M. Ren", A. B. $\operatorname{Rad}^{\#}$, W. L. Lo ${ }^{\#}$ and P. T. Chan \\ *Department of Automatic Control, Beijing Institute of Technology, Beijing 100081, China \\ ${ }^{*}$ Department of Electrical Engineering, The Hong Kong Polytechnic University, \\ Hung Hom, Kowloon, Hong Kong
}

\begin{abstract}
A new adaptive two degrees-of-freedom internal model control (IMC) with reference input predictor is presented for systems with unknown time delay. The proposed controller consists of a reference input predictor, a model reference controller, a disturbance rejection controller and an internal model predictor. The unknown time-delay and the rational transfer function of continuous-time systems are simultaneously estimated by using the modified least-squares with projection. One feature of the proposed algorithm is that its structure is integrated with an online parameter estimation algorithm to form an adaptive IMC with a reference input predictor. Simulation results demonstrate the effectiveness of the proposed control scheme. Copyright (C) 2005 IFAC
\end{abstract}

Keywords: Identification algorithms; Least-squares; Time delay estimation; Adaptive control.

\section{INTRODUCTION}

Control of time delay systems is difficult and challenging since the presence of a time delay in a control loop compels the system a sluggish response, detuned control action, and even instability of the controlled system. If the time delay is known, the celebrated Smith predictor (Smith, 1957) can successfully compensate the system by effectively removing the delay from the control loop. However, the performance heavily depends on the knowledge of a good model and the time delay of the system and consequently as a trade off, it is prone to mismatch models. Another popular control strategy that has been widely used to compensate time delay systems is the IMC control (Morari and Zafiriou, 1989). A close link between the IMC and the Smith predictor has been observed by many researchers. Laughlin et al. (1987) systematically analyzed the robustness of the Smith predictor within the IMC structure using several design methods. Zhang and $\mathrm{Xu}$ (2001) developed an analytical design method for the mismatched Smith predictor on the basis of the IMC structure.

The IMC structure (Morari and Zafiriou, 1989) uses the process model as a part of the controller and feedback is based on the difference between the outputs of the plant and its model. If the model matches the plant exactly, the IMC system becomes an open-loop system, and a perfect disturbance and tracking performance may be achieved. Recently, the IMC has shown a very popularity in process control industry due to its fine disturbance-rejection capabilities and robustness, as well as its simple yet effective framework for system design (Zhu et al., 1995; Lynch and Aalcudean, 1998; Wang et al., 2001; Zitek and Hlava, 2001). Since the IMC algorithm requires a model of the plant, an on-line identification is required to obtain the plant model when the plant is unknown or the plant parameters vary slowly due to aging.

In this paper, we consider the problem of designing and analyzing an IMC scheme for high-order systems with unknown time delay where not only the plant is identified on-line, but also the controller parameters are adapted to optimise the tracking error performance index such that good tracking to the output of the reference model and good disturbance rejection can be achieved simultaneously. This can be solved by designing a two degree-of-freedom IMC structure which decouples the disturbance response from the reference model tracking response.

The rest of this paper is organized as follows. Section 2 presents the two degree-of-freedom IMC with reference input predictor. Section 3 gives the identification model of time delay systems, followed by the modified least-squares with projection. Section 
4 evaluates the proposed algorithms with simulations. Section 5 concludes the paper.

\section{TWO DEGREE-OF-FREEDOM IMC WITH REFERENCE INPUT PREDICTOR}

Consider a linear time-invariant plant with time delay described by:

$$
\begin{aligned}
& y^{(n)}+a_{1} y^{(n-1)}+\cdots+a_{n} y \\
& \quad=b_{0} u^{(m)}(t-T)+b_{1} u^{(m-1)}(t-T)+\cdots+b_{m} u(t-T)
\end{aligned}
$$

where $T$ is the time delay; $a_{1}, \ldots, a_{n}, b_{0}, b_{1}, \ldots, b_{m}$ are plant parameters; $u(t), y(t)$ are the input and output of the plant, respectively. Taking Laplace transform on both sides of (1) with zero initial conditions, the transfer function of the plant (1) can be described by

$$
G(s)=\frac{B(s)}{A(s)} \exp (-T s)
$$

where $A(s)=s^{n}+a_{1} s^{n-1}+\cdots+a_{n}, B(s)=b_{0} s^{m}+b_{1} s^{m-1}+\cdots$ $+b_{m}$. Assume that the closed-loop reference model is $W_{m}(s)$, where $W_{m}(s)$ is a stable rational transfer function selected by the designers. Our objective is to design a controller so that the output $y$ of the controlled plant (1) asymptotically tracks the output $y_{m}$ of the stable reference model $W_{m}(s)$ for all piecewise continuous reference input signal $r(t)$. The IMC structure with model reference control is shown in Fig.1, where $C(s)$ is the controller, $\hat{G}(s)$ is the plant model, $d_{I}$ and $d_{o}$ are the bounded input and output disturbances, respectively.

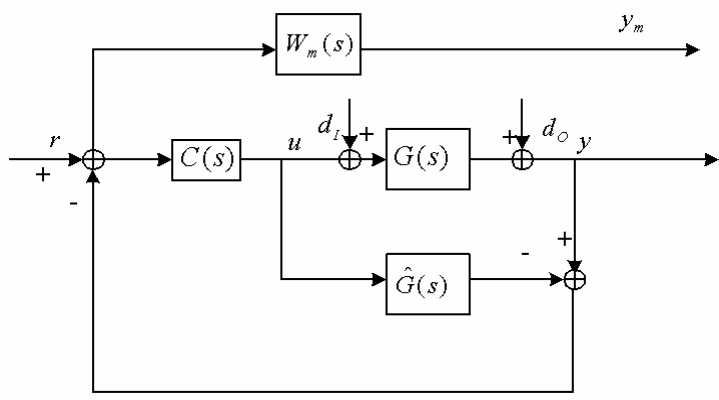

Fig.1 The IMC structure with model reference control.

Then the closed-loop transfer function is given by

$$
\begin{aligned}
y= & \frac{G(s) C(s)}{1+C(s)[G(s)-\hat{G}(s)]} r+\frac{1-\hat{G}(s) C(s)}{1+C(s)[G(s)-\hat{G}(s)]} d_{O} \\
& +\frac{G(s)[1-\hat{G}(s) C(s)]}{1+C(s)[G(s)-\hat{G}(s)]} d_{I} .
\end{aligned}
$$

It is easy to verify from (3) that, in the case of perfect modeling, the controller $C(s)=W_{m}(s) A(s) / B(s)$ gives the closed-loop transfer function described by

$y=W_{m}(s) e^{-T s} r+\left(1-W_{m}(s) e^{-T s}\right) d_{O}+G(s)\left(1-W_{m}(s) e^{-T s}\right) d_{I}$.

Obviously, there exists time delay response between the output of the stable reference model $W_{m}(s)$ and the plant output for the same time-varying reference input $r(t)$. In order to improve the tracking performance, prediction of future changes in the reference input is required. In addition, it can be seen from (4) that the effect of disturbances $d_{I}$ and $d_{o}$ on the plant output is determined by the reference model transfer function $W_{m}(s)$. Therefore, there is a compromise in selecting $W_{m}(s)$ for reference trajectory tracking and input and output disturbance rejections. In order to decouple the reference response from the input and output disturbance responses such that the disturbance responses can be improved without degrading the reference response, the two degree-of-freedom IMC with reference input predictor is shown in Fig.2, where $C_{r}(s)$ is the controller for the reference input $r(t), C_{d}(s)$ is the controller for the disturbances $d_{I}$ and $d_{o}$.

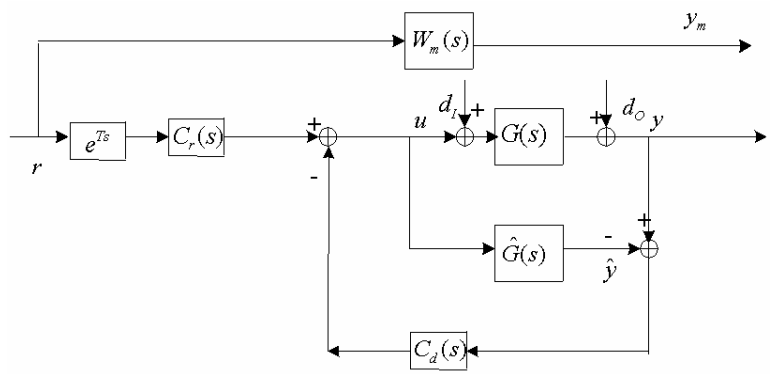

Fig.2 The two degree-of-freedom IMC with reference input predictor.

By $H_{2}$ optimal control, we can choose $C_{r}(s)$ and $C_{d}(s)$ to minimize the $L_{2}$ norm of the tracking error $y_{m}-y$ provided that $y_{m}-y \in L_{2}$. The minimum of the $H_{2}$ performance index can be achieved by selecting

$C_{r}(s)=W_{m}(s) \frac{A(s)}{B(s)}, \quad C_{d}(s)=\frac{A(s)}{B(s)}$.

This implies the control law is

$u=W_{m}(s) \frac{A(s)}{B(s)} r(t+T)+\frac{A(s)}{B(s)}(y-\hat{y}) \cdot$

The controller (6) is an algebraically optimal formulation, but it may become unstable if $B(s)$ contains the right-half-plane roots. To avoid these problems, let $z_{l}, z_{2}, \ldots, z_{l}$ be the open right half plane roots of $B(s)$ and define the Blashcke product (Datta, 1998)

$B^{+}(s)=\frac{\left(-s+z_{1}\right)\left(-s+z_{2}\right) \cdots\left(-s+z_{l}\right)}{\left(s+z_{1}^{-}\right)\left(s+z_{2}^{-}\right) \cdots\left(s+z_{l}^{-}\right)}$

where the symbol $\mathrm{z}^{-}$represents complex conjugation. In addition, we can write $B(s) / A(s)$ as

$\frac{B(s)}{A(s)}=B^{+}(s) \frac{B^{m}(s)}{A^{m}(s)}$

where $B^{m}(s) / A^{m}(s)$ is minimum phase. In order to make the optimal controllers $C_{r}(s)$ and $C_{d}(s)$ 
realizable, we may introduce filters $F_{r}, F_{d}$ to make $C_{r}(s)$ and $C_{d}(s)$ proper. In general, the filters $F_{r}, F_{d}$ are chosen as

$$
F_{r}(s)=\frac{1}{\left(\gamma_{r} s+1\right)^{n_{1}}}, F_{d}(s)=\frac{1}{\left(\gamma_{d} s+1\right)^{n_{2}}}
$$

where $\gamma_{r}, \gamma_{d}>0, n_{1}, n_{2}$ are chosen to be large enough positive integers to make $C_{r}(s)$ and $C_{d}(s)$ proper, respectively. Therefore, we can design the controllers $C_{r}(s), C_{d}(s)$ as

$$
C_{r}(s)=W_{m}(s) \frac{A^{m}(s)}{B^{m}(s)} F_{r}(s), C_{d}(s)=\frac{A^{m}(s)}{B^{m}(s)} F_{d}(s) .
$$

Once the controllers $C_{r}(s)$ and $C_{d}(s)$ are obtained, from (6), the control input to be implemented is derived by

$u=W_{m}(s) \frac{A^{m}(s)}{B^{m}(s)} F_{r}(s) r(t+T)+\frac{A^{m}(s)}{B^{m}(s)} F_{d}(s)(y-\hat{y})$.

\section{PLANT IDENTIFICATION}

In order to implement two degree-of-freedom IMC with reference input predictor, we need to design a parameter estimator to provide online estimates of the unknown parameters $a_{1}, \ldots, a_{n}, b_{0}, b_{1}, \ldots, b_{m}, T$ based on measures of input and output data. Considering the higher derivatives of input and output are not easily available for measurement, we filter both sides of (1) with $1 / \Lambda(s)$, where $\Lambda(s)=s^{n}+\lambda_{1} s^{n-1}+\cdots+\lambda_{n}$ is a stable Hurwitz polynomial, to obtain

$$
\begin{aligned}
\frac{s^{n}}{\Lambda(s)}[y]+a_{1} & \frac{s^{n-1}}{\Lambda(s)}[y]+\cdots+a_{n} \frac{1}{\Lambda(s)}[y]=b_{0} \frac{s^{m} \exp (-T s)}{\Lambda(s)}[u] \\
& +b_{1} \frac{s^{m-1} \exp (-T s)}{\Lambda(s)}[u]+\cdots+b_{m} \frac{\exp (-T s)}{\Lambda(s)}[u] .
\end{aligned}
$$

Adding $y$ to both sides of (12) and re-arranging the terms, we can obtain the parametric model for the plant (1) described by

$$
y=\theta^{T} \phi(T)
$$

where

$\theta=\left[\lambda_{1}-a_{1}, \lambda_{2}-a_{2}, \cdots, \lambda_{n}-a_{n}, b_{0}, b_{1}, \cdots, b_{m}\right]^{T}$

$\phi(T)=\left[\frac{s^{n-1}}{\Lambda(s)}[y], \cdots, \frac{1}{\Lambda(s)}[y], \frac{s^{m} \exp (-T s)}{\Lambda(s)}[u], \cdots, \frac{\exp (-T s)}{\Lambda(s)}[u]\right]^{T}$.

Let $\hat{\theta}, \hat{T}$ be the estimates of $\theta, T$, respectively. By (13), the estimate $\hat{y}$ of $y$ is formed by

$\hat{y}=\hat{\theta}^{T} \phi(\hat{T})$.

The identification error is defined by $e=y-\hat{y}$. Now we consider the generalized parameter vector $\hat{\Theta}=\left[\hat{\theta}^{T}, \hat{T}\right]^{T}$, the negative gradient of the identification error $e$ with respect to the generalized parameter $\hat{\Theta}$ is obtained by

$$
\Phi(\hat{\Theta})=-\frac{\partial e}{\partial \hat{\Theta}}=\left[\phi^{T}(\hat{T}),-\sum_{i=0}^{m} \hat{b}_{i} \frac{s^{m-i+1} \exp (-\hat{T} s)}{\Lambda(s)}[u]\right]^{T} .
$$

To update the parameter $\hat{\Theta}$, we consider the following cost function with a forgetting factor $\beta \geq 0$

$$
\begin{aligned}
J(\hat{\Theta}, t)= & \frac{1}{2} \int_{0}^{t} e^{-\beta(t-\tau)} \frac{\left[y(\tau)-\hat{\theta}^{T} \phi(\tau, \hat{T})\right]^{2}}{1+\gamma \Phi^{T}(\tau, \hat{\Theta}) \Phi(\tau, \hat{\Theta})} d \tau \\
& +\frac{1}{2} e^{-\beta t}\left(\hat{\Theta}-\hat{\Theta}_{0}\right)^{T} Q_{0}\left(\hat{\Theta}-\hat{\Theta}_{0}\right)
\end{aligned}
$$

where $\gamma \geq 0, Q_{0}=Q_{0}^{T}, \hat{\Theta}_{0}=\hat{\Theta}(0)$. The cost function (17) includes discounting of past data and a penalty on the initial estimate $\hat{\Theta}_{0}$ of $\hat{\Theta}$. To obtain the recursive formula for the parameter $\hat{\Theta}$, we transform the integral form of the cost function into a differential form by taking partial derivatives with respect to time

$$
\frac{\partial J(\hat{\Theta}, t)}{\partial t}+\beta J(\hat{\Theta}, t)=\frac{1}{2} \frac{\left[y(t)-\hat{\theta}^{T} \phi(t, \hat{T})\right]^{2}}{1+\gamma \Phi^{T}(t, \hat{\Theta}) \Phi(t, \hat{\Theta})} .
$$

Then, the partial derivative of $\partial J(\hat{\Theta}, t) / \partial t$ with respect to the parameter $\hat{\Theta}$ is given by

$$
\frac{\partial^{2} J(\hat{\Theta}, t)}{\partial \hat{\Theta} \partial t}+\beta \frac{\partial J(\hat{\Theta}, t)}{\partial \hat{\Theta}}=-\frac{e(t) \Phi(t, \hat{\Theta})}{1+\gamma \Phi^{T}(t, \hat{\Theta}) \Phi(t, \hat{\Theta}}-\frac{\gamma \frac{\partial \Phi}{\partial \hat{\Theta}} \Phi(t, \hat{\Theta}) e^{2}(t)}{\left[1+\gamma \Phi^{T}(t, \hat{\Theta}) \Phi(t, \hat{\Theta})\right]^{2}} .
$$

The total derivative of $\partial J(\hat{\Theta}, t) / \partial \hat{\Theta}$ with respect to time is given by

$\frac{d}{d t}\left[\frac{\partial J(\hat{\Theta}, t)}{\partial \hat{\Theta}}\right]=\frac{\partial^{2} J(\hat{\Theta}, t)}{\partial t \partial \hat{\Theta}}+\frac{\partial^{2} J(\hat{\Theta}, t)}{\partial^{2} \hat{\Theta}} \dot{\hat{\Theta}}$.

Substituting (19) into (20) yields

$$
\begin{aligned}
\frac{d}{d t}\left[\frac{\partial J(\hat{\Theta}, t)}{\partial \hat{\Theta}}\right]= & -\beta \frac{\partial J(\hat{\Theta}, t)}{\partial \hat{\Theta}}-\frac{e(t) \Phi(t, \hat{\Theta})}{1+\gamma \Phi^{T}(t, \hat{\Theta}) \Phi(t, \hat{\Theta})} \\
& -\frac{\gamma \frac{\partial \Phi}{\partial \hat{\Theta}} \Phi(t, \hat{\Theta}) e^{2}(t)}{\left[1+\gamma \Phi^{T}(t, \hat{\Theta}) \Phi(t, \hat{\Theta})\right]^{2}}+\frac{\partial^{2} J(\hat{\Theta}, t)}{\partial^{2} \hat{\Theta}} \dot{\hat{\Theta}} .
\end{aligned}
$$

The recursive least-squares is to estimate $\hat{\Theta}$ which minimizes the cost function (17) at each time interval for $t \geq 0$, that is, $\partial J(\hat{\Theta}, t) / \partial \hat{\Theta}=0$ for $\forall t \geq 0$. Substituting $\partial J(\hat{\Theta}, t) / \partial \hat{\Theta}=0$ into (21) gives the updating law for the parameter $\hat{\Theta}$

$$
\dot{\hat{\Theta}}=P \frac{e(t) \Phi(t, \hat{\Theta})}{1+\gamma \Phi^{T}(t, \hat{\Theta}) \Phi(t, \hat{\Theta})}+P \frac{\gamma \frac{\partial \Phi}{\partial \hat{\Theta}} \Phi(t, \hat{\Theta}) e^{2}(t)}{\left[1+\gamma \Phi^{T}(t, \hat{\Theta}) \Phi(t, \hat{\Theta})\right]^{2}} .
$$

where $P=\left[\partial^{2} J(\hat{\Theta}, t) / \partial \hat{\Theta}^{2}\right]^{-1}$.It follows from (17) that 


$$
\begin{aligned}
& \frac{\partial^{2} J(\hat{\Theta}, t)}{\partial \hat{\Theta}^{2}}=e^{-\beta t} Q_{0} \\
& +\int_{0}^{t} e^{-\beta(t-\tau)}\left(A_{1}(\tau, \hat{\Theta})+A_{2}(\tau, \hat{\Theta})+\gamma A_{3}(\tau, \hat{\Theta})\right) d \tau \\
& +\int_{0}^{t} e^{-\beta(t-\tau)} \gamma A_{2}(\tau, \hat{\Theta})\left[-A_{2}(\tau, \hat{\Theta})-4 A_{1}(\tau, \hat{\Theta})+4 \gamma A_{1}(\tau, \hat{\Theta}) A_{2}(\tau, \hat{\Theta})\right] d \tau
\end{aligned}
$$

where

$$
\begin{aligned}
& A_{1}(\tau, \hat{\Theta})=\frac{\Phi(\tau, \hat{\Theta}) \Phi^{T}(\tau, \hat{\Theta})}{1+\gamma \Phi^{T}(\tau, \hat{\Theta}) \Phi(\tau, \hat{\Theta})}, \\
& A_{2}(\tau, \hat{\Theta})=-\frac{e(\tau) \frac{\partial \Phi(\tau, \hat{\Theta})}{\partial \hat{\Theta}}}{1+\gamma \Phi^{T}(\tau, \hat{\Theta}) \Phi(\tau, \hat{\Theta})}, \\
& A_{3}(\tau, \hat{\Theta})=-\frac{\frac{\partial^{2} \Phi(\tau, \hat{\Theta})}{\partial \hat{\Theta}^{2}} \Phi(\tau, \hat{\Theta}) e^{2}(\tau)}{\left[1+\gamma \Phi^{T}(\tau, \hat{\Theta}) \Phi(\tau, \hat{\Theta})\right]^{2}} .
\end{aligned}
$$

Applying

the

identity $\frac{d}{d t} P P^{-1}=\dot{P} P^{-1}+P \frac{d}{d t} P^{-1}=0$, we can obtain that $P$ satisfies

$$
\begin{aligned}
\dot{P} & =\beta P-P\left[A_{1}(t, \hat{\Theta})+A_{2}(t, \hat{\Theta})+\gamma A_{3}(t, \hat{\Theta})\right. \\
& \left.+\gamma A_{2}(t, \hat{\Theta})\left(-A_{2}(t, \hat{\Theta})-4 A_{1}(t, \hat{\Theta})+4 \gamma A_{1}(t, \hat{\Theta}) A_{2}(t, \hat{\Theta})\right)\right] P \\
& -P\left[\int_{0}^{t} e^{-\beta(t-\tau)} \frac{d}{d t}\left(A_{1}(\tau, \hat{\Theta})+A_{2}(\tau, \hat{\Theta})+\gamma A_{3}(\tau, \hat{\Theta})\right) d \tau\right. \\
& \left.+\int_{0}^{t} e^{-\beta(t-\tau)} \frac{d}{d t}\left(\gamma A_{2}(\tau, \hat{\Theta})\left(-A_{2}(\tau, \hat{\Theta})-4 A_{1}(\tau, \hat{\Theta})+4 \gamma A_{1}(\tau, \hat{\Theta}) A_{2}(\tau, \hat{\Theta})\right)\right) d \tau\right] P
\end{aligned}
$$

where $P(0)=Q_{0}^{-1}$. Define the reference identification error as $\bar{e}(t)=e^{2}(t) /\left(1+\gamma \Phi^{T}(t, \hat{\Theta}) \Phi(t, \hat{\Theta})\right)$. Noting that

$$
\begin{aligned}
& \frac{\partial^{2} \bar{e}(\tau)}{\partial \hat{\Theta}^{2}}=A_{1}(\tau, \hat{\Theta})+A_{2}(\tau, \hat{\Theta})+\gamma A_{3}(\tau, \hat{\Theta}) \\
& +\gamma A_{2}(\tau, \hat{\Theta})\left[-A_{2}(\tau, \hat{\Theta})-4 A_{1}(\tau, \hat{\Theta})+4 \gamma A_{1}(\tau, \hat{\Theta}) A_{2}(\tau, \hat{\Theta})\right]
\end{aligned}
$$

we have

$$
\begin{aligned}
& \frac{d}{d t}\left(A_{1}(\tau, \hat{\Theta})+A_{2}(\tau, \hat{\Theta})+\gamma A_{3}(\tau, \hat{\Theta})+\gamma A_{2}(\tau, \hat{\Theta})\right. \\
& \left.\quad \times\left[-A_{2}(\tau, \hat{\Theta})-4 A_{1}(\tau, \hat{\Theta})+4 \gamma A_{1}(\tau, \hat{\Theta}) A_{2}(\tau, \hat{\Theta})\right]\right)=\frac{\partial^{3} \bar{e}(\tau)}{\partial \hat{\Theta}^{3}} \dot{\hat{\Theta}}
\end{aligned}
$$

Suppose that the estimated parameter $\hat{\Theta}$ is initialized in the vicinity of the actual value $\Theta$, we can use the second order Taylor series expansion to write

$$
\begin{aligned}
\bar{e}(\hat{\Theta})= & \bar{e}(\Theta)+\left.[\hat{\Theta}-\Theta]^{T} \frac{\partial \bar{e}}{\partial \hat{\Theta}}\right|_{\hat{\Theta}=\Theta} \\
& +\left.[\hat{\Theta}-\Theta]^{T} \frac{\partial^{2} \bar{e}}{\partial \hat{\Theta}^{2}}\right|_{\hat{\Theta}=\Theta}[\hat{\Theta}-\Theta]
\end{aligned}
$$

where the higher order terms in the Taylor series expansion have been dropped. Then, $\partial^{3} \bar{e} / \partial \hat{\Theta}^{3}=0$ can be obtained directly from (27). Furthermore, if the optimal estimate $\hat{\Theta}$ of $\Theta$ is unique, then we can conclude that $\frac{\partial^{2} e^{2}(t)}{\partial^{2} \hat{\Theta}}=\Phi(t, \hat{\Theta}) \Phi^{T}(t, \hat{\Theta})-e(t) \frac{\partial \Phi(t, \hat{\Theta})}{\partial \hat{\Theta}} \geq 0$ is satisfied for any $\hat{\Theta}$ in the vicinity of $\Theta$, which implies that

$1+\gamma \Phi^{T}(t, \hat{\Theta}) \Phi(t, \hat{\Theta})>\left[\gamma \Phi(t, \hat{\Theta}) \Phi^{T}(t, \hat{\Theta})\right]_{j j} \geq\left[\gamma \frac{\partial \Phi(t, \hat{\Theta})}{\partial \hat{\Theta}} e(t)\right]_{i j}$ is true for all elements $i, j=1, \cdots, n+m+2$. Therefore, the simplified least-squares can be described as

$$
\begin{aligned}
& \dot{\hat{\Theta}}=P \Phi e_{1}(t) \\
& \dot{P}=\beta P-P \frac{\Phi \Phi^{T}}{1+\gamma \Phi^{T} \Phi} P+P \frac{\partial \Phi}{\partial \hat{\Theta}} e_{1}(t) P
\end{aligned}
$$

where $e_{1}(t)=e(t) /\left(1+\gamma \Phi^{T}(t, \hat{\Theta}) \Phi(t, \hat{\Theta})\right)$.

Remark: Although the modified least-squares shown in (28) is an approximate algorithm, it can avoid the computational complexity for simultaneously estimating time delay and rational transfer function. If time delay $T$ is known, the generalized parameter vector $\hat{\Theta}$ and gradient vector $\Phi$ are the parameter vector $\hat{\theta}$ and gradient vector $\phi$, respectively. The derivative of $\phi$ with respect to the parameter $\hat{\theta}$ is equal to zero, the algorithm (28) corresponds to the conventional recursive normalized least-squares algorithm (Ioannou and Sun, 1996).

Define $\Omega_{1}$ to be a constraint set for $\hat{\Theta}$, that is, $\Omega_{1}=\left\{\hat{\Theta}:\|\hat{\Theta}\| \leq M_{1}, M_{1}>0\right\}$. We use the parameter gradient algorithm with projection to constraint the parameters $\hat{\Theta}$ within the set $\Omega_{1}$. The algorithm (28) can be modified by

$\dot{\hat{\Theta}}=\left\{\begin{array}{l}P \Phi e_{1}, \quad \text { if }\|\hat{\Theta}\|<M_{1} \text { or }\|\hat{\Theta}\|=M_{1} \text { and } \hat{\Theta}^{T} P \Phi e_{1} \leq 0 \\ P \Phi e_{1}-\frac{P \hat{\Theta} \hat{\Theta}^{T}}{\hat{\Theta}^{T} P \hat{\Theta}} P \Phi e_{1}, \quad \text { if }\|\hat{\Theta}\|=M_{1} \text { and } \hat{\Theta}^{T} P \Phi e_{1}>0\end{array}\right.$

and

$\dot{P}=\left\{\begin{array}{r}\beta P-P \frac{\Phi \Phi^{T}}{1+\gamma \Phi^{T} \Phi} P+P \frac{\partial \Phi}{\partial \hat{\Theta}} e_{1} P, \text { if }\|\hat{\Theta}\|<M_{1} \\ \text { or }\|\hat{\Theta}\|=M_{1} \text { and } \hat{\Theta}^{T} P \Phi e_{1} \leq 0 \\ 0, \text { if }\|\hat{\Theta}\|=M_{1} \text { and } \hat{\Theta}^{T} P \Phi e_{1}>0 .\end{array}\right.$

By the definition of the parameter $P, P$ is a positive definite matrix when the estimated parameters are initialized to some values in the vicinity of the actual values. Since $P=P^{T}>0$ and $\hat{\Theta}^{T} P \hat{\Theta}>0$ when $\|\hat{\Theta}\|=M_{1}$, no division by zero occurs in (29). The following theorem summarizes the properties of the modified least-squares with projection. 
Theorem: The adaptive law with projection algorithm described by (29) and (30) for the parametric model (15) with the input and output disturbances $d_{I}$ and $d_{O}$ guarantees that

(1) $\hat{\Theta}, P \in L_{\infty}$.

(2) For any $T \in(0, \infty)$, there exist constants $c_{1}, c_{2}$ such that the normalized identification error satisfies

$$
\int_{0}^{T} e_{1}^{2}(\xi)\left(1+\gamma \Phi^{T}(\xi) \Phi(\xi)\right) d \xi \leq c_{1}+c_{2} \int_{0}^{T} \frac{d^{2}(\xi)}{1+\gamma \Phi^{T}(\xi) \Phi(\xi)} d \xi
$$

where $d$ depends on the disturbances $d_{I}$ and $d_{O}$.

Proof. Due to space limitation, the proof is not included.

\section{SIMULATION}

In this section, we demonstrate the performance of the adaptive two degree-of-freedom IMC with reference input predictor with simulations and also compare the proposed algorithm with the IMC with model reference control and the Smith predictor under same conditions.

Example: Consider the following plant with dominant time delay

$$
y(s)=\frac{1.3}{s+0.8} e^{-10 s} u(s)
$$

where $a_{1}=0.8, b_{0}=1.3, T=10$ are parameters to be estimated. The reference model $W_{m}(s)$ is given by

$$
W_{m}(s)=\frac{1}{3 s+1}
$$

The reference input signal is a square waves with an amplitude of 1 and a period of 80 . Since the relative degree of the reference model $W_{m}(s)$ is equal to that of the rational dynamics in the plant (32), it is not necessary to use the filter $F_{r}(s)$ for the model reference controller $C_{r}(s)$. The disturbance rejection filter $F_{d}(s)$ can be chosen to be of the relative degree 1 to make $C_{d}(s)$ proper. In the simulation, we choose $F_{d}(s)=1 /(2.5 s+1)$, the stable Hurwitz polynomial $\Lambda(s)=s+0.5 \quad$, the estimator initial value $\hat{\Theta}=\left[\hat{a}_{1}(0), \hat{b}_{0}(0), \hat{T}(0)\right]^{T}=[1,1,8]^{T} \quad, \quad$ forgetting factor $\beta=0.03$ and $P(0)=\operatorname{diag}(1,1,1)$. A normal distribution noise signal with mean zero and variance of 0.01 as the output disturbance is added to the system output.

To compare the performance of the proposed control scheme with the Smith predictor, we use the adaptive PI control scheme suggested by Haalman (1965) in which PI controller parameters are selected based on the system model. Fig. 3 shows the simulation results of output tracking. Fig. 4 shows the corresponding estimated parameters $\hat{a}_{1}, \hat{b}_{0}, \hat{T}$. From Fig.3, we can observe that the adaptive two degree-of-freedom IMC with reference input predictor performs best performance over the adaptive IMC with model reference control and the Smith predictor for timevarying reference trajectory tracking. This is because the proposed control scheme includes the reference input predictor that can compensate for time delay transfer function between time-varying reference input and the plant output. These simulations also demonstrate that the modified least-squares algorithm with projection is effective for estimating unknown system parameters including time delay.

For comparison in the disturbance rejection capabilities of the three different control methods, a unit step set-point change is introduced for the plant (32) with the estimated parameters at $t=0$, and a $10 \%$ step disturbance is introduced at the plant input at $t=30$. The responses are shown in Fig.5. It can be seen that the proposed control scheme provides the best disturbance rejection over the IMC with model reference control and the Smith predictor.

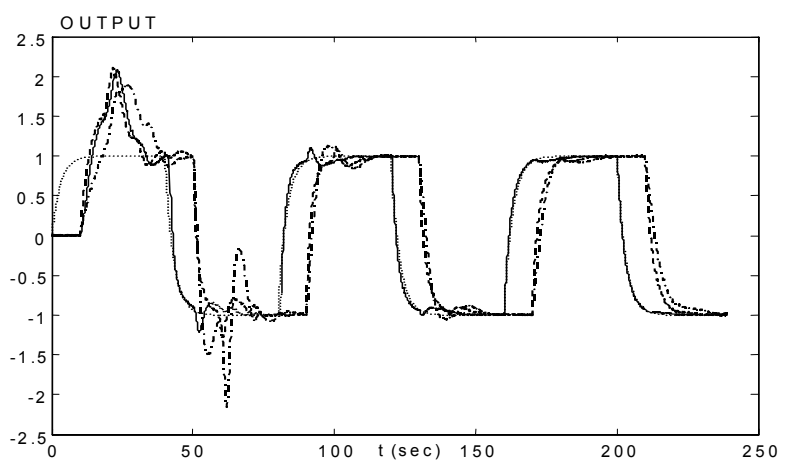

Fig.3. Output responses of the plant (32) (dotted line: reference model; solid line: proposed controller; dashed line: adaptive IMC; dashed-dotted line: adaptive Smith predictor).

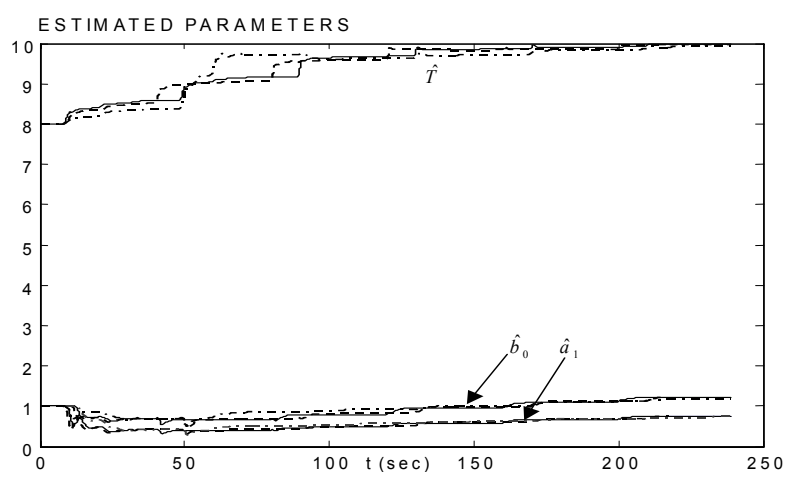

Fig.4. Parameter estimates of the plant (32) (solid line: proposed controller; dashed line: adaptive IMC; dashed-dotted line: adaptive Smith predictor). 


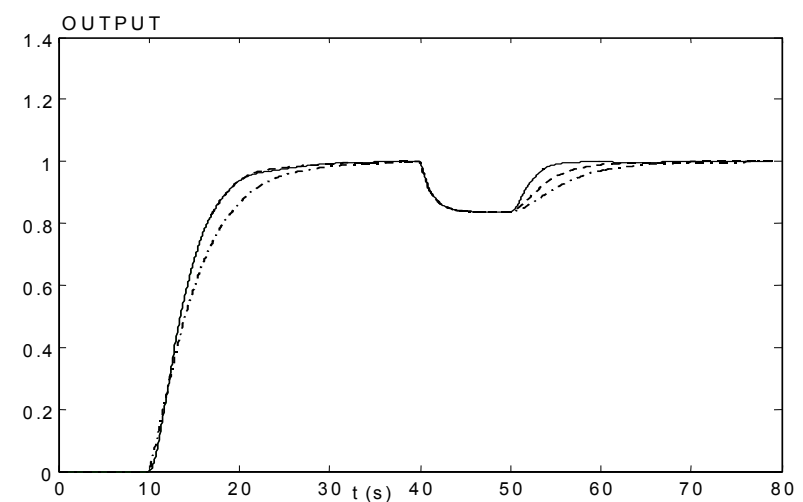

Fig. 5. Disturbance responses of the plant (32) (solid line: proposed controller; dashed line: IMC; dashed-dotted line: Smith predictor).

\section{CONCLUSION}

The adaptive control of a two degree-of-freedom IMC with reference input predictor is introduced and its stability property is investigated. Simulated examples show that two degree-of-freedom IMC with reference input predictor is more suitable for the control of time delay systems in the presence of noise than the IMC with model reference control and the Smith predictor.

\section{ACKNOWLEDGEMENT}

The authors gratefully acknowledge the financial support of The Hong Kong Polytechnic University through the grant no.G-U020. The first author also acknowledges the financial support of the National Natural Science Foundation of China through the grant no.60474033.

\section{REFERENCES}

Datta, A.(1998). Adaptive internal model control, Berlin: Springer.

Haalman, A. (1965). Adjusting controllers for deadtime processes. Control Engineering, 12,7175.

Ioannou, P. A. and J. Sun (1996). Robust adaptive control, Upper Saddle River, N.J.: PTR PrenticeHall.

Laughlin, D. L., D.E. Rivera and M. Morari (1987). Smith predictor design for robust performance. Int. J. Control, 46(2), 477-504.

Lynch, A.F. and S.E. Aalcudean (1998). Internal model based controller design using measured costs and gradients. Int. J. Control, 69(2), 257270.

Morari, M. and E. Zafiriou (1989). Robust process control, Englewood Cliffs, NJ: Prentice-Hall.

Pintelon, R. and J. Schoukens (2001). System identification: a frequency domain, IEEE Press.

Smith, O. J. M. (1957). Closer control of loops with dead time. Chemical Engineering, 53(5), 217-219.
Wang Q. G., Q. Bi and Y. Zhang (2001). Partial internal model control. IEEE Trans. on Industrial Electronics, 48(5), 976-982.

Zhang, W. and X. Xu (2001). Analytical design and analysis of mismatched Smith predictor. IST Transactions, 40, 133-138.

Zhu, H. A., C.L. Teo , A. N. Poo and G. S. Hong (1995). An enhanced internal model structure. Control Theory Adv. Technol., 10, 1115-1127.

Zitek, P. and J. Hlava (2001). Anisochronic internal model control of time-delay systems. Control Engineering Practice, 9, 501-516. 